\title{
Preclinical Evaluation of Dual mTOR Inhibitor, AZD2014, in Prostate Cancer
}

\author{
Chiranjeevi Sandi ${ }^{1}$, Antonio Ramos-Montoya ${ }^{1}$, Sergio L. Filisbino ${ }^{2}$, Sarah Jurmeister ${ }^{1}$, Madhu \\ Basetti ${ }^{1}$, Karan Wadhwa ${ }^{1}$, John R. Griffiths ${ }^{1}$, Frances M. Richards ${ }^{1}$, Duncan I. Jodrell ${ }^{1}$, David \\ E. Neal ${ }^{1,3}$, Sabina Cosulich ${ }^{4}$, Barry R. Davies ${ }^{5}$ and Simon Pacey ${ }^{1,6}$

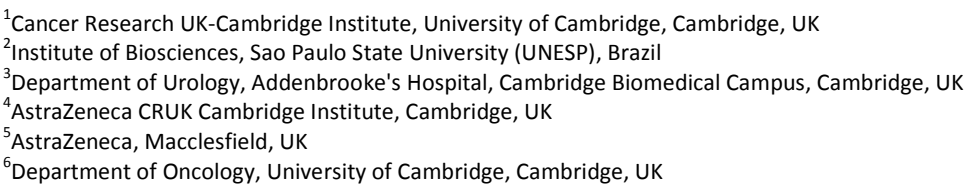

Background: An estimated 220,800 cases and 27,540 deaths from prostate cancer (PCa) will occur in the USA during 2015. Altered PI3K/AKT/mTOR signalling contributes to prostate cancer progression and transition to androgen-independent disease, for example one study reported $42 \%$ of primary and $100 \%$ of metastatic PCa tumours exhibited mutations, altered expression or copy number variations within this pathway. First generation mTOR inhibitors (preferentially inhibit mTORC1), have had limited anti-cancer effect in patients with $\mathrm{PCa}$, possibly due to negative feedback activation of the AKT pathway via mTORC2. The dual mTORC1/2 inhibitor, AZD2014, may overcome this liability. Using a genetically engineered PTEN conditional mouse model (Pten ${ }^{\text {loxp/loxp }}$;PB-Cre4), we have investigated the effects of AZD2014. The studies complement a clinical trial (NCT02064608) of AZD2014, given to men before radical prostatectomy and are timed for when invasive prostate carcinomas develop in the model around 10-14 months prior to onset of resistance to castration through AKT pathway activation. AZD2014, 15mg/kg daily, oral (with or without castration) or vehicle were administered for 14 days.

Results: AZD2014 was well tolerated with no overt toxicity observed. Pharmacokinetic (PK) analysis revealed mean concentrations of $4.4 \pm 2.1 \mu \mathrm{M}$ of AZD2014 in the plasma samples collected 4 hours after day 14 dose. AZD2014 alone or combined with castration inhibited mTORC1 and mTORC2, measured by reductions in p4EBP1(Thr37/46) by approximately $48 \%(p<0.001)$ and $37 \%(p<0.001)$; pS6(Ser235/236) by $74 \%(p<0.001)$ and $44 \%(p<0.001)$ and pAKT(Ser473) by $36 \%(p<0.001)$ and $20 \%$ $(p<0.01)$ as compared to vehicle-treated mice. AZD2014 treatment was anti-proliferative; Ki67 was significantly reduced in AZD2014-treated mice $(70 \%, p<0.001)$ or AZD2014 plus castration (42\%, $p<0.001)$. Apoptosis was detected with cleaved caspase 3 and increased by 3.3 -fold $(p<0.001)$ in both AZD2014 or AZD2014 plus castration groups and 2 -fold $(p<0.001)$ in the castration only group, respectively. Tumour volumes (ultrasound imaging) were reduced by $51 \%(p<0.05)$ comparing AZD2014 plus castration against control. HRMAS ${ }^{1} \mathrm{H}$ NMR spectroscopy was used on tumour tissue to determine changes in metabolites following treatment and identified that the total choline to creatine ratio ( $\mathrm{t}$-Cho/ $\mathrm{Cr}$ ) was reduced by $40 \%$ in AZD2014-treated mice tumour samples $(\mathrm{p}<0.05)$ as compared to control-treated mice.

Conclusions: Short term (14 days) treatment with AZD2014 with or without castration was associated with both pharmacodynamic and anti-tumour effects. The total choline/creatine ratio, previously reported as positively correlated with Gleason score in PCa patients, might be, in addition to our standard mTOR PD markers, utilised as a non-invasive biomarker of AZD2014 activity. The primary and phenotypic biomarker effects of monotherapy with AZD2014 in this relevant genetically engineered mouse model of prostate cancer will be compared with paired biopsies from the ongoing exploratory window study in the prostate cancer patients prior to prostatectomy, and may inform potential novel combination approaches that are translatable to the clinic. 